
\title{
25 Research Soure \\ Positive Correlation Between the Hip Adduction Moment Impulse During the Stance Phase and the Hip Joint Contact Force Impulse
}

\section{Takuma Inai ( $\sim$ hwd17001@nuhw.ac.jp )}

Institute for Human Movement and Medical Sciences, Niigata University of Health and Welfare https://orcid.org/0000-0001-8806-7389

\section{Tomoya Takabayashi}

Institute for Human Movement and Medical Sciences, Niigata University of Health and Welfare

Mutsuaki Edama

Institute for Human Movement and Medical Sciences, Niigata University of Health and Welfare

\section{Masayoshi Kubo}

Institute for Human Movement and Medical Sciences, Niigata University of Health and Welfare

\section{Research}

Keywords: Walking, hip, joint contact force, moment impulse

Posted Date: December 17th, 2020

DOl: https://doi.org/10.21203/rs.3.rs-127175/v1

License: (c) (i) This work is licensed under a Creative Commons Attribution 4.0 International License. Read Full License 


\section{Abstract}

Background: Excessive mechanical loading, in the form of the joint contact force, has been reported to promote osteoarthritis in vitro and vivo in mice. However, it has also been reported that an excessive hip adduction moment impulse during the stance phase likely contributes to the progression of hip osteoarthritis. The relationship between the hip adduction moment impulse and hip joint contact force (impulse, and first and second peaks) during the stance phase is unclear. The objective of the present study was to clarify this relationship.

Methods: A public dataset pertaining to the overground walking of 84 healthy adults, in which the participants walked at a self-selected speed, was considered. The data of three trials for each participant were analyzed. The relationship between the hip adduction moment and hip joint contact force, in terms of the impulse and first and second peaks, during the stance phase was evaluated using correlation coefficients.

Results: The hip adduction moment impulse during the stance phase was positively correlated with the hip joint contact force impulse and not correlated with the first and second peak hip joint contact forces. Furthermore, the first and second peak hip adduction moments during the stance phase were positively correlated with the first and second peak hip joint contact forces, respectively.

Conclusions: These findings indicate that the hip joint contact force impulse during the stance phase can be used as an index to determine the risk factors for the progression of hip osteoarthritis.

\section{Introduction}

Hip osteoarthritis leads to hip joint pain [1] and a decrease in the muscle strength [2,3] and range of hip joint motions $[4,5]$. Moreover, this condition leads to a deterioration in the patients' ability to perform activities of daily living [6,7] and consequently, the quality of life [8]. Therefore, it is necessary to identify the risk factors for the progression of hip osteoarthritis to effectively prevent this progression.

A previous study [9] indicated that excessive mechanical loading in the form of the joint contact force promotes osteoarthritis in vitro and vivo in mice. Furthermore, in another study [10], researchers identified a risk factor for hip osteoarthritis, that is, the excessive daily cumulative hip moment in the frontal plane, which is the product of the hip adduction moment impulse and mean steps per day. However, the hip adduction moment impulse, which is the time integration of the hip adduction moment, is not the same as the hip joint contact force. Therefore, in this study, the hip adduction moment impulse during the stance phase was considered as a risk factor for the progression of hip osteoarthritis.

Nevertheless, the components of the hip joint contact force during the stance phase (i.e., impulse, first peak, or second peak) that constitute a risk factor for the progression of hip osteoarthritis are unclear. With reference to the previously reported findings [10], a definitive risk factor can be identified by 
examining the relationship between the hip adduction moment impulse and each component of the hip joint contact force (i.e., impulse, first peak, and second peak) during the stance phase.

To this end, the purpose of the present study was to examine the relationship between the hip adduction moment and hip joint contact force, in terms of the respective impulses, first peaks, and second peaks during the stance phase. We hypothesized that the hip adduction moment impulse during the stance phase is positively correlated with the hip joint contact force impulse, and that the hip adduction moment impulse is not correlated with the first and second peak hip joint contact forces.

\section{Methods}

\section{Participants}

A public dataset for the overground walking data of 300 participants, which was reported in a previous study [11], was used. To determine the sample size required to attain a reasonable power for the correlation analysis, an a priori power analysis was conducted using $\mathrm{R}$ Studio (power $=0.8$, significance level $=.05$, effect size $=0.3$ [medium] [12]). The results indicated that the appropriate number of participants was 84 . Furthermore, according to a previous study [13], the mean age of patients suffering from hip osteoarthritis is more than $40 \mathrm{y}$. Therefore, the overground walking data of 84 adults aged between 40 and 69 y were selected randomly and used in the analyses. Table 1 lists the characteristics of all the participants. The study protocol was approved by the ethics committee of the National Institute of Advanced Industrial Science and Technology [11].

Table 1

Participant characteristics $(n=84)$.

\begin{tabular}{|lllll|}
\hline & Mean & SD & Minimum & Maximum \\
\hline Age, years & 55.5 & 8.7 & 40 & 68 \\
\hline Sex & 42 males and 42 females & $\square$ & $\square$ & $\square$ \\
\hline Height, $\mathrm{m}$ & 1.63 & 0.09 & 1.38 & 1.81 \\
\hline Body mass, $\mathrm{kg}$ & 61.0 & 10.3 & 34 & 89 \\
\hline Body mass index & 22.8 & 2.9 & 17.3 & 31.5 \\
\hline
\end{tabular}

\section{Experimental protocol and data acquisition}

The details of the experimental protocol and data acquisition can be found in the existing report [11]. Briefly, data acquisition was performed in a room with a straight $10 \mathrm{~m}$ path for the participants to walk on [11]. Three-dimensional position data of 55 to 59 reflective markers attached to the participants' body landmarks and the ground reaction forces were obtained using a 3D motion-capture-system (VICON MX, 
UK) with a sampling frequency of $200 \mathrm{~Hz}$ and force plates (AMTI, USA) sampled at $1000 \mathrm{~Hz}$ [11]. The participants were asked to walk barefoot at a comfortable, self-selected speed [11]. Prior to the experiment, the participants were allowed to perform sufficient practice walks to ensure the maintenance of the natural gait [11]. After the practice, the data for 10 gait cycles (five from the right leg and five from the left leg), as determined by the force plates, were recorded [11].

\section{Data processing}

For each participant, three trials for the left lower limb were selected randomly and analyzed. We analyzed the stance phase of the left limb (i.e., from the left heel contact to the left toe-off) for each participant, according to a previous study [10]. Furthermore, the three-dimensional marker trajectories, ground reaction forces, center of pressure, and moments of the force plates were filtered using a fourthorder Butterworth low-pass filter at a cut-off frequency of $6 \mathrm{~Hz}$, as described in a previous study [14].

The external hip adduction moment during the stance phase of the left limb for each participant was calculated considering the inverse dynamics (Newton-Euler method). Subsequently, the hip adduction moment impulse was calculated by the integration of the hip adduction moment. The hip adduction moment impulse (Nms) was normalized using the body mass $(\mathrm{Nms} / \mathrm{kg})$, as described in an existing report [15]. The first and second peak hip adduction moments $(\mathrm{Nm})$ were calculated at the first $(0-50 \%)$ and second halves (50-100\%) of the stance phase, respectively, and normalized using the body mass $(\mathrm{Nm} / \mathrm{kg})$.

The masses, mass positions, and inertia parameters of the segments, as reported in previous studies $[16,17]$, were employed. The hip joint center was determined according to the method proposed by Hara et al. [18]. In particular, the knee joint center was defined as the midpoint of the medial and lateral epicondyles of the femur. The ankle joint center was defined as the midpoint of the medial and lateral malleoli. All the gait analyses were conducted using Scilab.

\section{Musculoskeletal model}

A musculoskeletal model was established, according to the findings of a previous study [19]. The number of degrees of freedom (DoF) of the musculoskeletal model was 11 (the pelvis [6 DoF], hip [3 DoF], knee [1 DoF], and ankle [1 DoF]), and the number of muscles was 55 [19]. The muscles were represented using the Hill model and consisted of a contractile, passive, and series element for each muscle [20]. The forcelength and force-velocity relationships for the contractile elements were established [21]. In general, the passive elements for each muscle generate a passive force when the muscle is lengthened [21]. However, according to a previous study [22], the passive hip joint moment is small when the range of hip joint is from approximately extension $10^{\circ}$ to flexion $30^{\circ}$, such as in the case of walking (i.e., the stance phase [23]). Therefore, the passive force for each muscle was neglected. The musculoskeletal model was scaled for each participant considering the leg lengths of each participant and a cadaver [19].

\section{Muscle force and hip joint contact force}


To estimate the muscle forces during the stance phase, static optimization (to minimize the square of the muscle activation [24,25]) was performed. Furthermore, the hip joint contact force during the stance phase was calculated based on a previous study [25]. The hip joint contact force impulse was calculated by integrating the hip joint contact force during the stance phase. The hip joint contact force $(\mathrm{N})$ was normalized using the body mass $(\mathrm{Ns} / \mathrm{kg})$. The first and second peak hip joint contact forces $(\mathrm{N})$ were calculated at the first $(0-50 \%)$ and second halves $(50-100 \%)$ of the stance phase, respectively, and normalized using the body mass $(\mathrm{N} / \mathrm{kg})$.

\section{Statistics}

The Shapiro-Wilk test was conducted to determine whether the variables (hip adduction moment impulse, first and second hip adduction moments, hip joint contact force impulse, and first and second peak hip joint contact forces) followed a normal distribution. Based on the results of the normality, Pearson's correlation or Spearman's correlation was used. The significance level was set as $<0.05$. As described in a previous study [12], the effect size (Large: $0.5<$, Medium: $0.3-0.5$, Small: $0.1-0.3$, and Negligible: <0.1) was determined for each correlation.

\section{Results}

Table 2 lists the correlation coefficients for the hip adduction moment (impulse, first peak, and second peak) and hip joint contact force (impulse, first peak, and second peak) during the stance phase. The hip adduction moment impulse and hip joint contact force impulse during the stance phase exhibited a significantly positive correlation. Moreover, the first peak hip adduction moment and hip joint contact force, and the second peak hip adduction moment and hip joint contact force were significantly positively correlated. The first peak hip adduction moment and hip joint contact force impulse, and the second peak hip adduction moment and hip joint contact force impulse, were significantly positively correlated.

Table 2

Correlation coefficients for external hip adduction moment and hip joint contact force during walking.

\begin{tabular}{|c|c|c|c|}
\hline & $\begin{array}{l}\text { Hip joint contact } \\
\text { force impulse, } \\
\text { Ns } / \mathrm{kg}\end{array}$ & $\begin{array}{l}\text { First peak hip joint } \\
\text { contact force, } \\
\mathrm{N} / \mathrm{kg}\end{array}$ & $\begin{array}{l}\text { Second peak hip } \\
\text { joint contact force, } \\
\text { N/kg }\end{array}$ \\
\hline $\begin{array}{l}\text { Hip adduction moment impulse, } \\
\mathrm{Nms} / \mathrm{kg}\end{array}$ & $\begin{array}{l}r=0.58(\text { Large }), p \\
<0.001\end{array}$ & $\begin{array}{l}r=0.06 \\
\text { (Negligible), } p= \\
0.576\end{array}$ & $\begin{array}{l}r=0.20 \text { (Small), } p= \\
0.064\end{array}$ \\
\hline $\begin{array}{l}\text { First peak external hip } \\
\text { adduction moment, Nm/kg }\end{array}$ & $\begin{array}{l}r=0.34 \text { (Medium), } \\
p=0.001\end{array}$ & $\begin{array}{l}r=0.57 \text { (Large), } p< \\
0.001\end{array}$ & $\begin{array}{l}r=0.18 \text { (Small), } p= \\
0.110\end{array}$ \\
\hline $\begin{array}{l}\text { Second peak external hip } \\
\text { adduction moment, } \mathrm{Nm} / \mathrm{kg}\end{array}$ & $\begin{array}{l}r=0.42 \text { (Medium), } \\
p<0.001\end{array}$ & $\begin{array}{l}r=-0.01 \\
\text { (Negligible), } p= \\
0.929\end{array}$ & $\begin{array}{l}r=0.52 \text { (Large), } p< \\
0.001\end{array}$ \\
\hline
\end{tabular}


Figures $1 \mathrm{a}$ and $1 \mathrm{~b}$ show the waveform of the averaged external hip adduction moment $(\mathrm{Nm} / \mathrm{kg})$ and averaged hip joint contact force $(\mathrm{N} / \mathrm{kg})$ during the stance phase, respectively. In both the waveforms, the first and second peaks occurred at approximately $20 \%$ and $80 \%$ of the stance phase, respectively.

Table 3 summarizes the results of the kinetics during the stance phase, specifically, the mean and standard deviation (SD) for each variable.

Table 3

Kinetic parameters during walking.

\begin{tabular}{|lll|}
\hline & Mean & SD \\
\hline Hip adduction moment impulse, Nms/kg & 0.27 & 0.05 \\
\hline First peak external hip adduction moment, Nm/kg & 0.82 & 0.14 \\
\hline Second peak external hip adduction moment, Nm/kg & 0.62 & 0.12 \\
\hline Hip joint contact force impulse, Ns/kg & 16.7 & 2.4 \\
\hline First peak hip joint contact force, N/kg & 40.2 & 8.3 \\
\hline Second peak hip joint contact force, N/kg & 48.0 & 8.0 \\
\hline
\end{tabular}

\section{Discussion}

In this study, the relationship between the hip adduction moment and hip joint contact force, in terms of the impulse, first peak, and second peak, during the stance phase was examined. The main findings were as follows: (1) The hip adduction moment impulse during the stance phase is positively correlated with the hip joint contact force impulse; however, the hip adduction moment impulse is not correlated with the first and second peak hip joint contact forces; (2) the positive correlation coefficient for the hip adduction moment impulse and hip joint contact force impulse during the stance phase is large compared to that for the first (or second) peak hip adduction moment and the hip joint contact force impulse during the stance phase; and (3) the first and second peak hip adduction moments are positively correlated with the first and second peak hip joint contact forces, respectively.

Some researchers [26] examined the relationships between the hip joint moments and first (or second) peak hip joint contact force during the stance phase. Furthermore, in several recent studies $[14,15,27]$, the hip adduction moment impulse during the stance phase was examined. However, the relationship between the hip adduction moment impulse and hip joint contact force impulse during the stance phase has not been clarified yet. This study represents the first attempt to examine the relationship between the hip adduction moment impulse and hip joint contact force impulse during the stance phase, and the presented findings may provide valuable insight to understand the relationships between the hip 
adduction moment and hip joint contact force, in terms of the impulse, first peak, and second peak, during the stance phase.

As mentioned previously, it has been reported that an excessive mechanical loading in the form of the joint contact force promotes osteoarthritis in vitro and vivo in mice [9]. Moreover, another report [10] indicated that a high daily cumulative hip moment in the frontal plane is a risk factor for the progression of hip osteoarthritis. The results in the present study indicate that a high hip joint contact force impulse, and not the first and second peaks, during the stance phase may be a risk factor for the progression of hip osteoarthritis.

In particular, the mean (SD) hip adduction moment impulse during the stance phase was $0.27(0.05)$ $\mathrm{Nms} / \mathrm{kg}$, which is approximately similar to the values reported by Tateuchi et al. [10], specifically, 0.41 $(0.13) \mathrm{Nm} / \mathrm{kg}$. Moreover, the mean (SD) first peak hip adduction moment during the stance phase was $0.82(0.14) \mathrm{Nm} / \mathrm{kg}$ (Table 3), which is similar to that reported by Tateuchi et al. [10], specifically, 1.05 $(0.29) \mathrm{Nm} / \mathrm{kg}$. According to these values, the values obtained in the present study can be considered quantitatively reasonable.

Furthermore, the mean (SD) first and second peak hip joint contact forces during the stance phase were 40.2 (8.3) N/kg and 48.0 (8.0) N/kg, respectively. These values are slightly higher than the actual values measured using instrumented prostheses in previous studies [28-30]. According to the existing literature [26], it can be considered that the results are influenced by the differences in the parameters of the musculoskeletal model. However, because we used the same musculoskeletal model for each participant, our findings can be considered valid, even though the values are slightly higher than the actual values.

Nevertheless, this study involves certain limitations. It cannot be conclusively stated that a high hip joint contact force impulse during the stance phase is a risk factor for the progression of hip osteoarthritis because a prospective cohort study was not performed. It is thus necessary to perform a prospective cohort study in future work. Furthermore, in this study, the data of healthy adults who did not suffer from hip osteoarthritis were considered. However, it has been reported that [13] the patients with hip osteoarthritis exhibit different hip joint moments during walking compared to those of healthy participants. Therefore, the relationship between the hip adduction moment and hip joint contact force during the stance phase, in terms of the impulse, first peak, and second peak, must be examined considering the data of patients suffering from hip osteoarthritis.

\section{Conclusions}

The relationship between the hip adduction moment and hip joint contact force, in terms of the impulse, first peak, and second peak during the stance phase was examined. It was observed that the hip adduction moment impulse during the stance phase is positively correlated with the hip joint contact force impulse; however, the hip adduction moment impulse is not correlated with the first and second peak hip joint contact forces. Moreover, the positive correlation coefficient for the hip adduction moment impulse and hip joint contact force impulse during the stance phase is large compared to that between 
the first (or second) peak hip adduction moment and hip joint contact force impulse during the stance phase. It is thus considered that a high hip joint contact force impulse, and not the first and second peaks, during the stance phase may be a risk factor for the progression of hip osteoarthritis. These findings can provide valuable insight to conclusively define a risk factor for the progression of hip osteoarthritis.

\section{Declarations}

\section{Ethics approval and consent to participate}

Not applicable.

\section{Consent for publication}

Not applicable.

\section{Availability of data and material}

All files are available from AIST Gait Database 2019 (URL:

https://unit.aist.go.jp/harc/ExPART/GDB2019_e.html).

\section{Competing interests}

The authors declare that they have no competing interests.

\section{Funding}

None.

\section{Authors' contributions}

TI, TT, ME, and MK discussed the conception and design of this study. TI analyzed the data. TT, ME, and MK read the first manuscript written by $\mathrm{TI}$, and critically revised it. All authors approved the final version of the manuscript.

\section{Acknowledgements}

We would like to thank Editage (www.editage.jp) for the English language editing.

\section{Conflict of interest}

The authors declare no conflicts of interest.

\section{References}


1. lidaka T, Muraki S, Oka H, Horii C, Kawaguchi H, Nakamura K, et al. Incidence rate and risk factors for radiographic hip osteoarthritis in Japanese men and women: A 10-year follow-up of the ROAD study. Osteoarthr Cartil. W.B. Saunders Ltd; 2020;28:182-8.

2. Zacharias A, Pizzari T, English DJ, Kapakoulakis T, Green RA. Hip abductor muscle volume in hip osteoarthritis and matched controls. Osteoarthr Cartil. W.B. Saunders Ltd; 2016;24:1727-35.

3. Loureiro A, Mills PM, Barrett RS. Muscle weakness in hip osteoarthritis: A systematic review. Arthritis Care Res (Hoboken) [Internet]. 2013 [cited 2017 Jan 4];65:340-52. Available from: http://www.ncbi.nlm.nih.gov/pubmed/22833493

4. Holla JFM, Steultjens MPM, van der Leeden M, Roorda LD, Bierma-Zeinstra SMA, den Broeder AA, et al. Determinants of range of joint motion in patients with early symptomatic osteoarthritis of the hip and/or knee: An exploratory study in the CHECK cohort. Osteoarthr Cartil [Internet]. 2011 [cited 2017 Jan 4];19:411-9. Available from: http://www.ncbi.nlm.nih.gov/pubmed/21272657

5. Holla JFM, van der Leeden M, Roorda LD, Bierma-Zeinstra SMA, Damen J, Dekker J, et al. Diagnostic accuracy of range of motion measurements in early symptomatic hip and/or knee osteoarthritis. Arthritis Care Res (Hoboken) [Internet]. 2012 [cited 2017 May 5];64:59-65. Available from: http://www.ncbi.nlm.nih.gov/pubmed/21954179

6. Pisters MF, Veenhof C, van Dijk GM, Dekker J, CARPA Study Group. Avoidance of activity and limitations in activities in patients with osteoarthritis of the hip or knee: A 5 year follow-up study on the mediating role of reduced muscle strength. Osteoarthr Cartil [Internet]. 2014 [cited 2017 May 5];22:171-7. Available from: http://www.ncbi.nlm.nih.gov/pubmed/24333296

7. Pisters MF, Veenhof C, van Dijk GM, Heymans MW, Twisk JWR, Dekker J. The course of limitations in activities over 5 years in patients with knee and hip osteoarthritis with moderate functional limitations: Risk factors for future functional decline. Osteoarthr Cartil. Osteoarthritis Cartilage; 2012;20:503-10.

8. Boutron I, Rannou F, Jardinaud-lopez M, Meric G, Revel M, Poiraudeau S. Disability and quality of life of patients with knee or hip osteoarthritis in the primary care setting and factors associated with general practitioners' indication for prosthetic replacement within 1 year. Osteoarthr Cartil. Osteoarthritis Cartilage; 2008;16:1024-31.

9. Chang SH, Mori D, Kobayashi H, Mori Y, Nakamoto H, Okada K, et al. Excessive mechanical loading promotes osteoarthritis through the gremlin-1-NF-KB pathway. Nat Commun. Nature Publishing Group; 2019;10:1442.

10. Tateuchi H, Koyama Y, Akiyama H, Goto K, So K, Kuroda Y, et al. Daily cumulative hip moment is associated with radiographic progression of secondary hip osteoarthritis. Osteoarthr Cartil [Internet]. 2017 [cited 2017 Apr 7];25:1291-8. Available from: http://www.ncbi.nlm.nih.gov/pubmed/28232145

11. Kobayashi Y, Hida N, Nakajima K, Fujimoto M, Mochimaru M. AIST Gait Database 2019. 2019; Available from: https://unit.aist.go.jp/harc/ExPART/GDB2019_e.html

12. Cohen J. A power primer. Psychol Bull. 1992;112:155-9. 
13. Diamond LE, Allison K, Dobson F, Hall M. Hip joint moments during walking in people with hip osteoarthritis: A systematic review and meta-analysis. Osteoarthr Cartil [Internet]. 2018 [cited 2019 Mar 31];26:1415-24. Available from: http://www.ncbi.nlm.nih.gov/pubmed/29621605

14. Inai T, Takabayashi T, Edama M, Kubo M. Effect of contralateral cane use on hip moment impulse in the frontal plane during the stance phase. Gait Posture [Internet]. Elsevier; 2019 [cited 2019 Mar 31];70:311-6. Available from: https://www.sciencedirect.com/science/article/pii/S096663621930339X

15. Inai T, Takabayashi T, Edama M, Kubo M. Decrease in walking speed increases hip moment impulse in the frontal plane during the stance phase. PeerJ. PeerJ Inc.; 2019;7:e8110.

16. de Leva P. Adjustments to Zatsiorsky-Seluyanov's segment inertia parameters. J Biomech [Internet]. 1996 [cited 2016 Jun 12];29:1223-30. Available from:

http://www.ncbi.nlm.nih.gov/pubmed/8872282

17. Robertson DGE, Caldwell GE, Hamill J, Kamen G, Whittlesey SN. Research Methods in Biomechanics. 2nd ed. Champaign: Human Kinetics; 2013.

18. Hara R, McGinley J, Briggs C, Baker R, Sangeux M. Predicting the location of the hip joint centres, impact of age group and sex. Sci Rep [Internet]. 2016 [cited 2019 Mar 14];6:37707. Available from: http://www.ncbi.nlm.nih.gov/pubmed/27883044

19. Carbone V, Fluit R, Pellikaan P, van der Krogt MM, Janssen D, Damsgaard M, et al. TLEM 2.0 - A comprehensive musculoskeletal geometry dataset for subject-specific modeling of lower extremity. J Biomech. Elsevier Ltd; 2015;48:734-41.

20. Inai T, Takabayashi T, Edama M, Kubo M. Algorithm to compute muscle excitation patterns that accurately track kinematics using a hybrid of numerical integration and optimization. J Biomech [Internet]. Elsevier; 2020 [cited 2020 May 20];in press. Available from: https://linkinghub.elsevier.com/retrieve/pii/S0021929020302591

21. Zajac FE. Muscle and tendon: Properties, models, scaling, and application to biomechanics and motor control. Crit Rev Biomed Eng. 1989;17:359-411.

22. Riener R, Edrich T. Identification of passive elastic joint moments in the lower extremities. J Biomech. Elsevier Science Ltd; 1999;32:539-44.

23. Fukuchi CA, Fukuchi RK, Duarte M. A public dataset of overground and treadmill walking kinematics and kinetics in healthy individuals. PeerJ [Internet]. 2018 [cited 2019 Mar 31];6:e4640. Available from: http://www.ncbi.nlm.nih.gov/pubmed/29707431

24. Erdemir A, McLean S, Herzog W, van den Bogert AJ. Model-based estimation of muscle forces exerted during movements. Clin Biomech. 2007;22:131-54.

25. Inai T, Takabayashi T, Edama M, Kubo M. Effect of hip joint angle at seat-off on hip joint contact force during sit-to-stand movement: A computer simulation study 11 Medical and Health Sciences 1103 Clinical Sciences. Biomed Eng Online. BioMed Central Ltd.; 2018;17:177.

26. Wesseling M, de Groote F, Meyer C, Corten K, Simon JP, Desloovere K, et al. Gait alterations to effectively reduce hip contact forces. J Orthop Res [Internet]. 2015 [cited 2017 Apr 28];33:1094-102. 
Available from: http://www.ncbi.nlm.nih.gov/pubmed/25676535

27. Inai T, Takabayashi T, Edama M, Kubo M. Evaluation of factors that affect hip moment impulse during gait: A systematic review. Gait Posture [Internet]. Elsevier; 2018 [cited 2018 Mar 27];61:48892. Available from: http://www.ncbi.nlm.nih.gov/pubmed/29494823

28. Bergmann G, Deuretzbacher G, Heller M, Graichen F, Rohlmann A, Strauss J, et al. Hip contact forces and gait patterns from routine activities. J Biomech [Internet]. 2001;34:859-71. Available from: http://www.ncbi.nlm.nih.gov/pubmed/11410170

29. Stansfield BW, Nicol AC, Paul JP, Kelly IG, Graichen F, Bergmann G. Direct comparison of calculated hip joint contact forces with those measured using instrumented implants. An evaluation of a threedimensional mathematical model of the lower limb. J Biomech. 2003;36:929-36.

30. Modenese L, Phillips ATM. Prediction of hip contact forces and muscle activations during walking at different speeds. Multibody Syst Dyn. Springer; 2012;28:157-68.

\section{Figures}
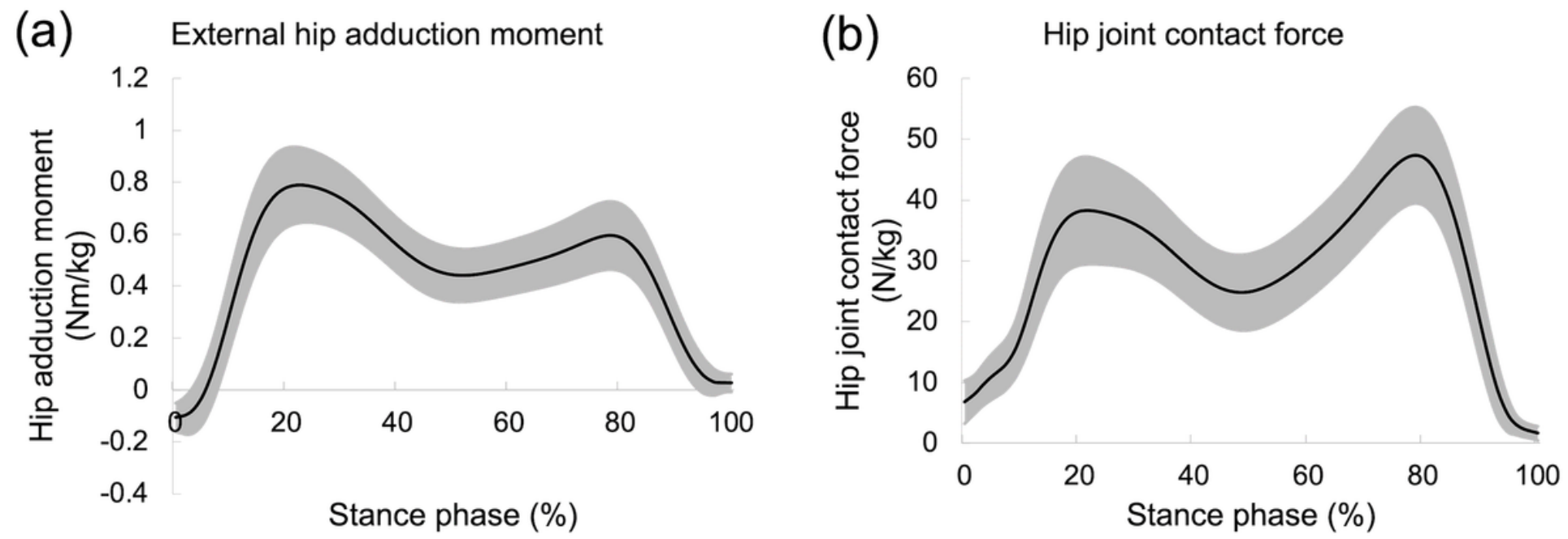

\section{Figure 1}

(a) External hip adduction moment and (b) hip joint contact force during the stance phase. Bimodal peaks were observed in both the waveforms. The gray shading indicates the standard deviation. 

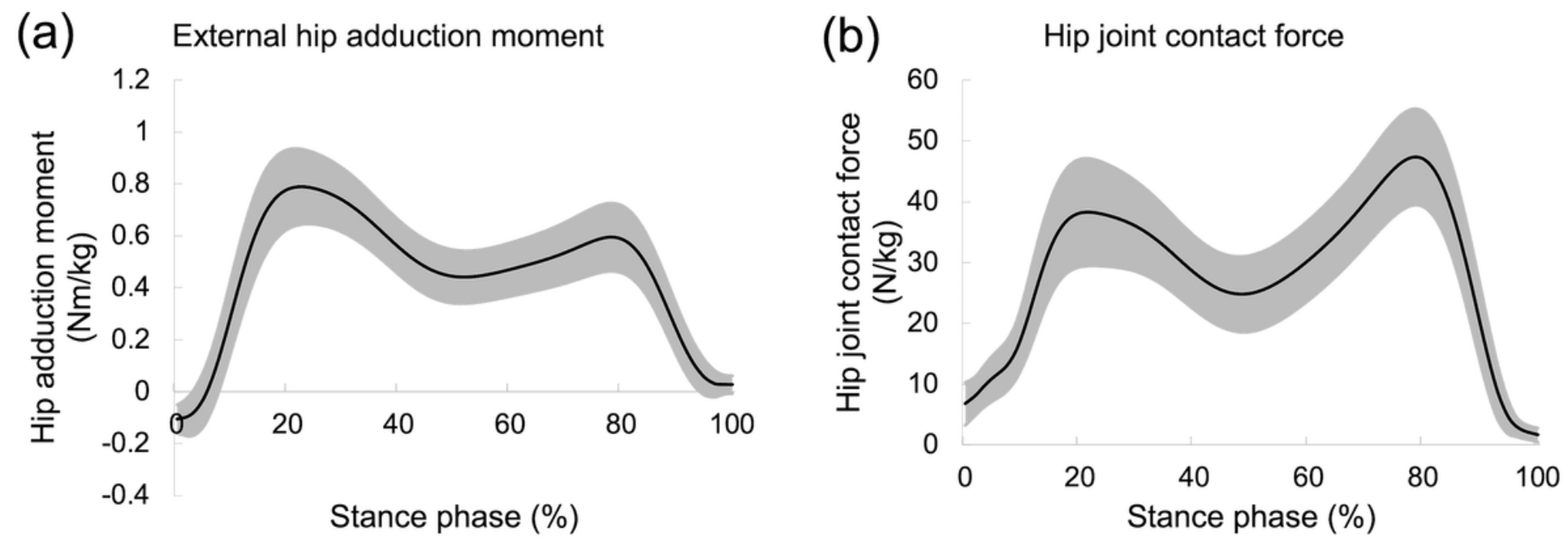

\section{Figure 1}

(a) External hip adduction moment and (b) hip joint contact force during the stance phase. Bimodal peaks were observed in both the waveforms. The gray shading indicates the standard deviation.
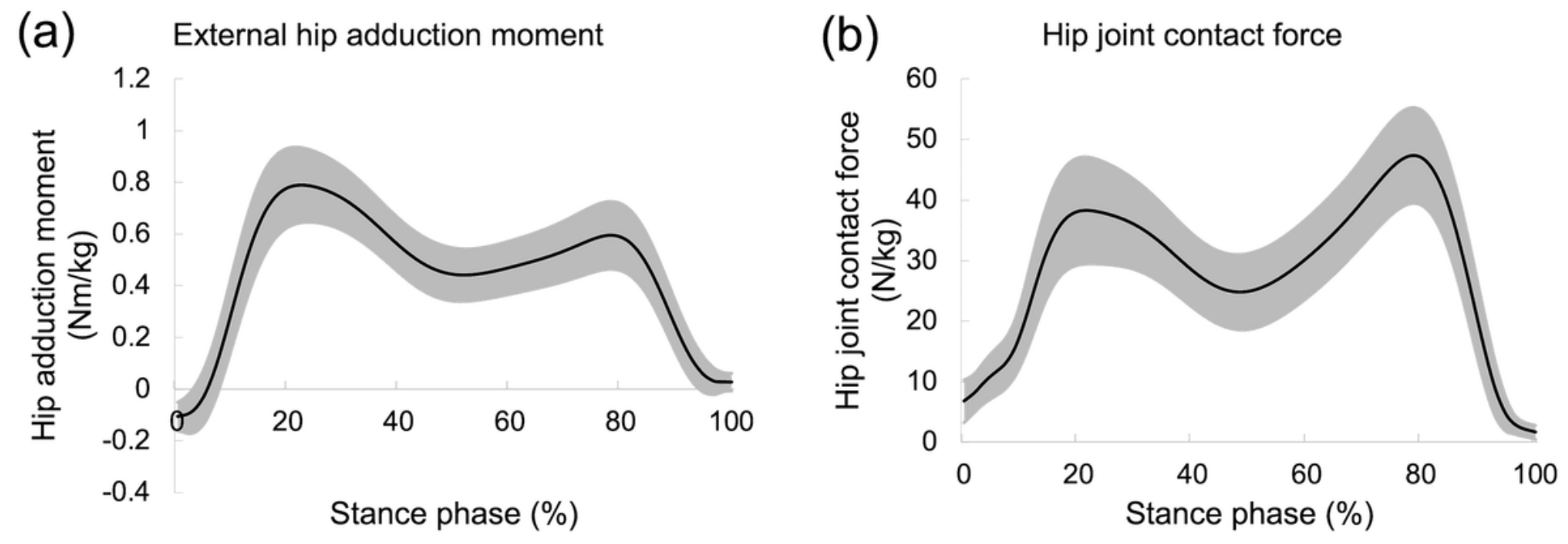

Figure 1

(a) External hip adduction moment and (b) hip joint contact force during the stance phase. Bimodal peaks were observed in both the waveforms. The gray shading indicates the standard deviation. 